\title{
Helical Partial Snake for the AGS
}

\author{
T. Roser, M. Syphers, E. Courant, L. Ratner, M. Okamura
}

March 17, 1998

The success of the Partial Siberian Snake in the AGS has paved the way to ever increasing polarization of proton beams at high energy, up to $50 \%$ polarization at $21 \mathrm{GeV}$ during the last polarized proton run. The latest improvement made toward reaching this level of performance was the introduction of an oscillating dipole field during acceleration. This magnet is used to induce a coherent betatron oscillation at a frequency near the betatron tune, enhancing the resonance strength and generating spin flip as the beam crosses four strong intrinsic resonances. It has been observed that much of the remaining depolarization occuring in the AGS is associated with transverse coupling resonances, whereby depolarizing resonances are seen not only when the vertical tune is at an integer relation with $G \gamma$, but when the horizontal tune is as well. And, in fact, a major source of the transverse coupling is the strong solenoidal field of the Partial Siberian Snake.

To combat the transverse coupling inherently produced by a solenoidal magnetic field, we have investigated the use of a helical dipole magnet in the AGS to generate a Partial Snake. Helical magnets will also generate some degree of transverse coupling, but it is shown below that the level of the coupling is dramatically reduced by this configuration. Unlike the helical dipole magnet Snakes to be used in RHIC, which each consist of four $2.4 \mathrm{~m}$ long superconducting, 4 Tesla magnets, the AGS Partial Snake would be composed of a single normal conducting helix with steering magnets on either side. The use of one helix with surrounding dipole magnets as a Snake has been proposed previously[1], but in the context of a full Snake (180 ${ }^{\circ}$ spin rotation). Here, we propose a solution where the spin rotation is some small fraction of $180^{\circ}$, and which fits in the allotted straight section space of the AGS.

\section{An AGS Helical Snake}

The orbital trajectory through a $360^{\circ}$ helical dipole magnet of central field strength $B_{0}$, length $L_{h}$, and pitch $k=2 \pi / L_{h}$ is given by

$$
x(s)=x_{0}+\frac{B_{0}}{k^{2}(B \rho)}[\cos (k s+\alpha)-\cos \alpha]+\left[x_{0}^{\prime}+\frac{B_{0}}{k(B \rho)} \sin (\alpha)\right] s
$$




$$
y(s)=y_{0}+\frac{B_{0}}{k^{2}(B \rho)}[\sin (k s+\alpha)-\sin \alpha]+\left[y_{0}^{\prime}-\frac{B_{0}}{k(B \rho)} \cos (\alpha)\right] s
$$

where $B \rho$ is the magnetic rigidity of the particle. Here, $\alpha$ is the angle with respect to vertical of the magnetic field at the beginning of the helical magnet. For a full helical magnet where the magnetic field begins and ends horizontally $(\alpha=-\pi / 2)$, the trajectory at the exit is given by

$$
\begin{aligned}
x\left(L_{h}\right) & =x_{0}+x_{0}^{\prime} L_{h}-\frac{B_{0} L_{h}}{k(B \rho)} \\
x^{\prime}\left(L_{h}\right) & =x_{0}^{\prime} \\
y\left(L_{h}\right) & =y_{0}+y_{0}^{\prime} L_{h} \\
y^{\prime}\left(L_{h}\right) & =y_{0}^{\prime}
\end{aligned}
$$

Thus, to minimize the orbit excursions within the Helix, we use bend magnets located a distance $L_{c h}$ upstream and downstream of the Helix which produce deflections $\pm \theta_{c}$ such that

$$
\theta_{c} L_{c h}+\theta_{c} L_{h}+\delta_{0}+\theta_{c} L_{c h}=0
$$

where $\delta_{0} \equiv-\frac{B_{0} L_{h}}{k(B \rho)}$ (see Fig. 1).

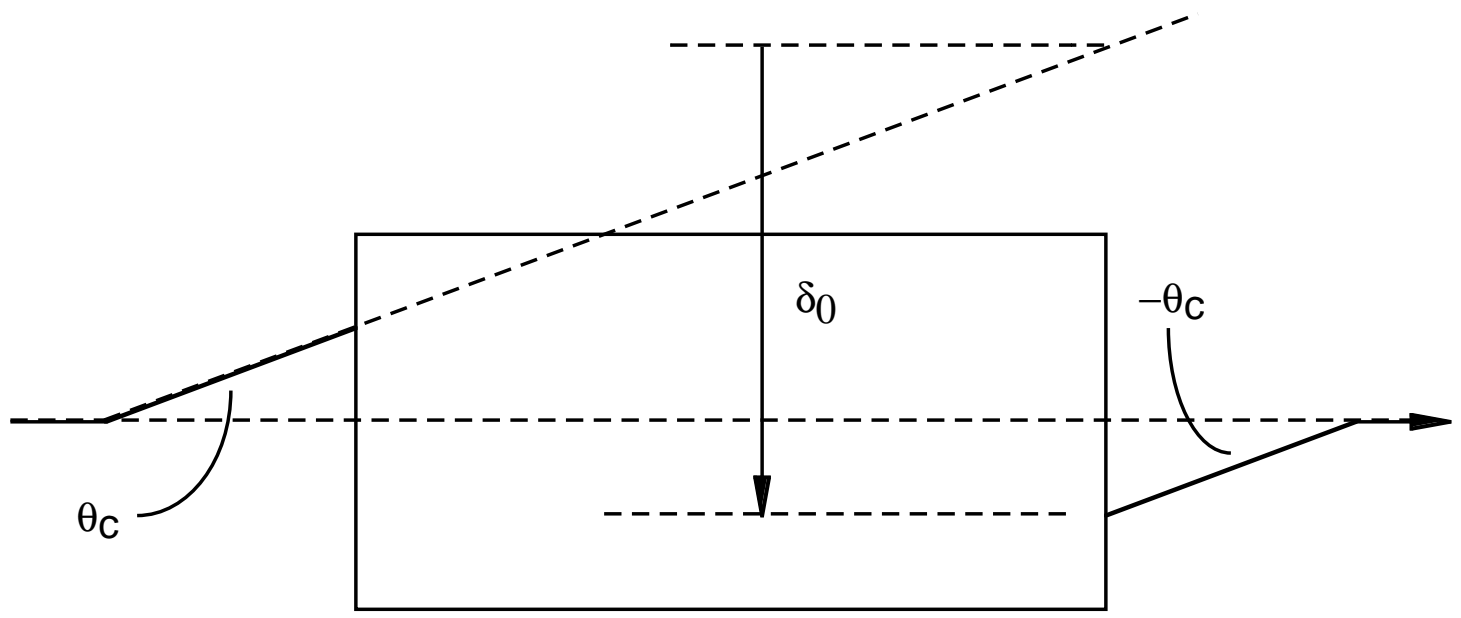

Figure 1: Dipole and Helix arrangement for Partial Snake in AGS.

We assume that the helical dipole field strength will be about 1.5 Tesla, and that the entire system will have to fit into a standard 10 foot straight section of the AGS. Chosing a helical dipole length of $L_{h}=1.5 \mathrm{~m}$, this gives $\delta_{0}=-36 \mathrm{~mm}$. We take bend centers of the bending magnets to be a distance $L_{c h}=50 \mathrm{~cm}$ from the ends of the Helix, and give the bending magnets lengths of $30 \mathrm{~cm}$. With these parameters, the bending magnets must have total integrated field strengths of 0.11 Tesla-meters, or $B_{c}$ 


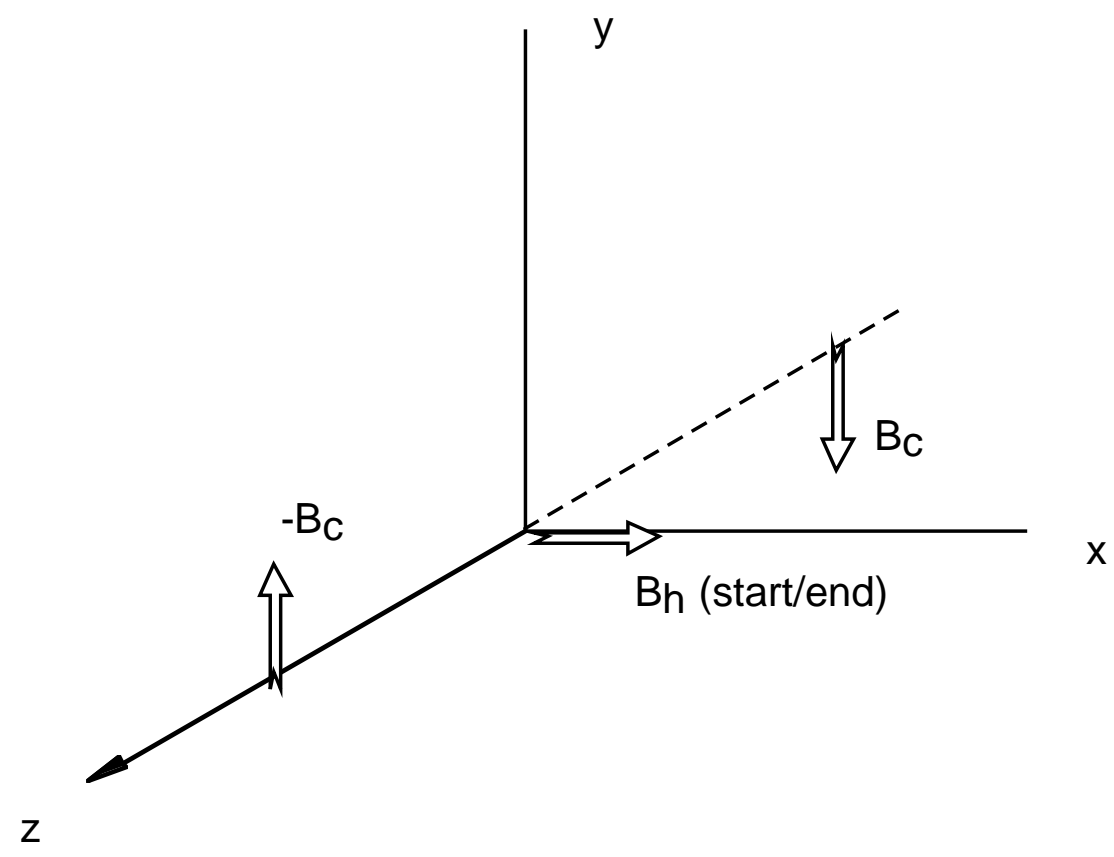

Figure 2: Field configuration for the Helical Snake.

$=0.72$ Tesla over the $30 \mathrm{~cm}$ magnetic length, with the arrangement of field polarities shown in Fig. 2. These parameters can be optimized further after considering actual mechanical designs. The maximum orbital offset in the system is approximately $20 \mathrm{~mm}$ in the horizontal plane, and $25 \mathrm{~mm}$ in the vertical plane, as seen in Fig. 3, at an injection total energy of $2.4 \mathrm{GeV}$. The excursions are reduced at higher energies, as the magnets must be held at constant field.

\section{Spin Motion through the Snake}

The spin matrices of the components of the Helical Snake can be written as follows. Acting upon a 3 -component spin vector, $\vec{S}^{T}=\left(S_{x}, S_{y}, S_{z}\right)$, the spin matrix for a bending magnet with horizontal orbit deflection is

$$
M_{d}(\theta)=\left(\begin{array}{ccc}
\cos \mu & 0 & -\sin \mu \\
0 & 1 & 0 \\
\sin \mu & 0 & \cos \mu
\end{array}\right)
$$


Trajectory through Helical Partial Snake

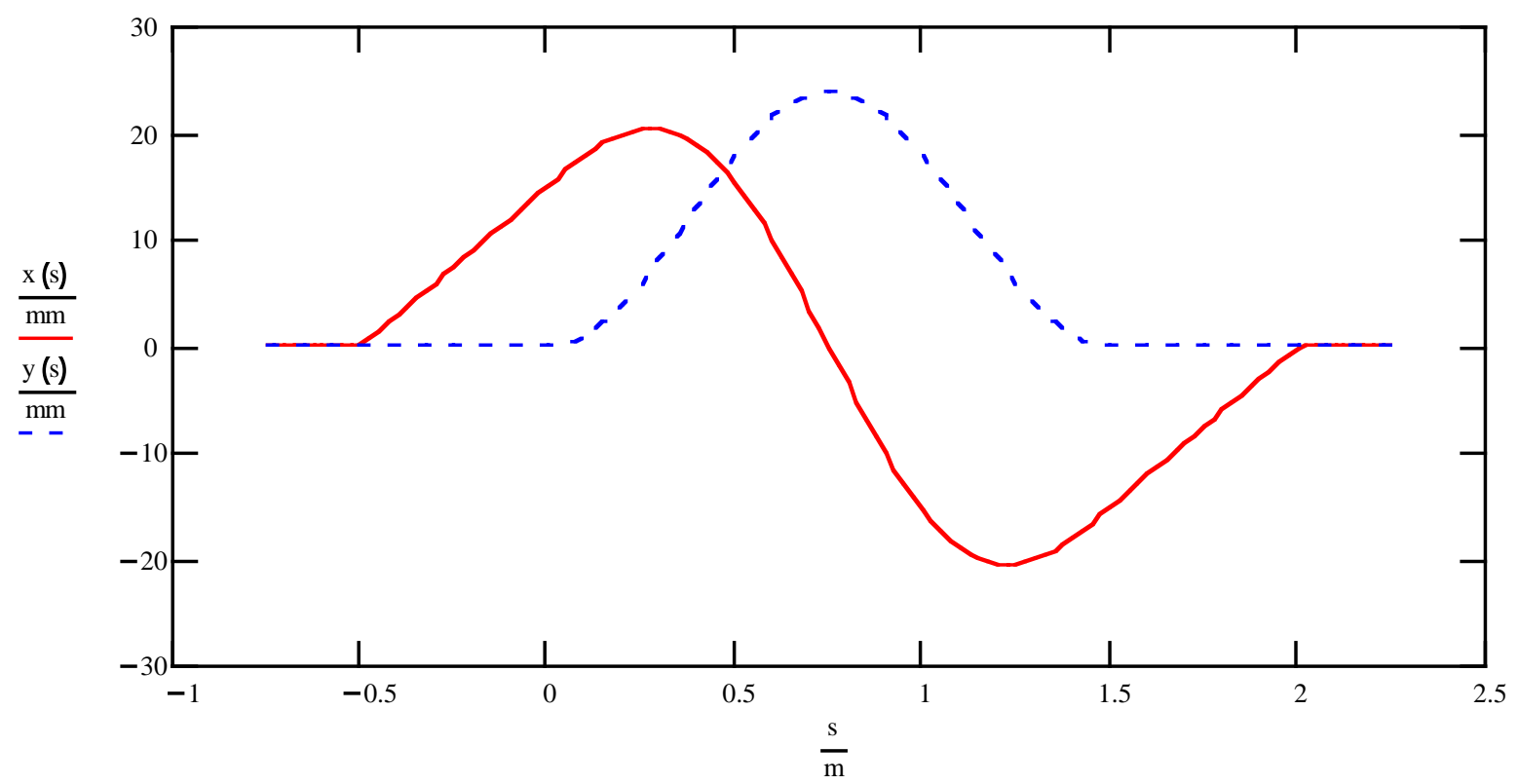

Figure 3: Trajectory through Helical Partial Snake in AGS. The origin, $s=0$, corresponds to the entry to the helical dipole magnet, which is $1.5 \mathrm{~m}$ long.

where $\mu \equiv(1+G \gamma) \theta, \theta$ being the bend angle of the magnet. Here, $\theta$ is positive for a negative vertical field component. For the helix, the spin matrix is

$$
M_{h}=\left(\begin{array}{ccc}
\cos ^{2} \phi \cos \mu+\sin ^{2} \phi & -\cos \phi \sin \mu & \cos \phi \sin \phi(1-\cos \mu) \\
\cos \phi \sin \mu & \cos \mu & -\sin \phi \sin \mu \\
\cos \phi \sin \phi(1-\cos \mu) & \sin \phi \sin \mu & \sin ^{2} \phi \cos \mu+\cos ^{2} \phi
\end{array}\right)
$$

where this time $\mu=-k L_{h} \sqrt{1+(K / k)^{2}}$, with $K \equiv(1+G \gamma) B_{0} /(B \rho)$, and $\phi=$ $\tan ^{-1}(K / d)$.

The total spin matrix for the helical dipole system would then be given by

$$
M_{\text {spin }}=M_{d}\left(-\theta_{c}\right) M_{h} M_{d}\left(\theta_{c}\right) .
$$

This matrix rotates the spin through an angle $\delta$ about an axis of rotation, $\vec{n}$. In general, if a $3 \times 3$ matrix $M$ is that of a rotation transformation, then the trace of the matrix is just

$$
\operatorname{tr} M=1+2 \cos \mu_{r},
$$

where $\mu_{r}$ is the angle of rotation. This most easily can be seen by noting that a suitable transformation of coordinates can be performed which identifies one of the three coordinate axes as the axis of rotation. Then, the resulting rotation matrix 
would be of the form

$$
M_{f}=U M U^{-1}=\left(\begin{array}{ccc}
\cos \mu_{r} & \sin \mu_{r} & 0 \\
-\sin \mu_{r} & \cos \mu_{r} & 0 \\
0 & 0 & 1
\end{array}\right)
$$

where $U$ performs the coordinate transformation. The above result is now obvious, since the trace of the resulting matrix would be the same as the trace of the original matrix, the trace being invariant under cyclic permutations of matrix multiplications.

To find the spin precession axis, $\vec{n}$, we can use the infinitesimal rotation generators

$$
\Delta_{1}=\left(\begin{array}{ccc}
0 & 0 & 0 \\
0 & 0 & -1 \\
0 & 1 & 0
\end{array}\right), \Delta_{2}=\left(\begin{array}{ccc}
0 & 0 & 1 \\
0 & 0 & 0 \\
-1 & 0 & 0
\end{array}\right), \quad \Delta_{3}=\left(\begin{array}{ccc}
0 & -1 & 0 \\
1 & 0 & 0 \\
0 & 0 & 0
\end{array}\right)
$$

given the rotation angle $\mu_{r}$. If the elements of the matrix $M$ are denoted by $m_{i j}$, then one notes that the trace of the product $\Delta_{i} M$ is just

$$
\operatorname{tr}\left(\Delta_{i} M\right)=m_{j k}-m_{k j}
$$

where $i j k$ form a cyclic set of indices and $\operatorname{tr} M$ stands for the trace of the matrix $M$. In general, the Euler parameters[2]

$$
\begin{aligned}
e_{0} & \equiv \cos \frac{\mu_{r}}{2} \\
\vec{e} & \equiv \vec{n} \sin \frac{\mu_{r}}{2}
\end{aligned}
$$

can be used to describe each element of the matrix $M$. Namely,[3]

$$
m_{i j}=2 e_{i} e_{j}+\sum_{k=1}^{3}\left[\delta_{i j}\left(e_{0}^{2}-e_{k} e_{k}\right)+\epsilon_{i j k} e_{0} e_{k}\right]
$$

where $\delta_{i j}=1$ if $i=j$ and zero otherwise, and $\epsilon_{i j k}=0$ if any two indices are equal, otherwise +1 if the indices are an even permutation, or -1 if the indices are an odd permutation of 123. Applying these definitions to $\operatorname{tr}\left(\Delta_{i} M\right)$ above, we find that the components of the unit vector along the direction of the rotation axis satisfy

$$
\operatorname{tr}\left(\Delta_{k} M\right)=2 n_{k} \sin \mu_{r}
$$

For our spin matrix $M_{\text {spin }}$ the spin precession angle is therefore

$$
\delta=\cos ^{-1}\left(\frac{\operatorname{tr} M_{\text {spin }}-1}{2}\right)
$$

and the axis of rotation is given by

$$
\vec{n}^{T}=\left(\frac{\operatorname{tr}\left(\Delta_{1} M_{\text {spin }}\right)}{2 \sin \delta}, \frac{\operatorname{tr}\left(\Delta_{2} M_{\text {spin }}\right)}{2 \sin \delta}, \frac{\operatorname{tr}\left(\Delta_{3} M_{\text {spin }}\right)}{2 \sin \delta}\right) .
$$


Putting in the parameters of our system described above, we find that the angle of rotation is $\delta=9.7$ degrees, or, in other words, the system generates a $5 \%$ Partial Siberian Snake. This is approximately the strength used in the present solenoid Partial Snake in the AGS. The spin rotation axis of the system, however, is not exactly in the longitudinal direction as it is in the solenoid system. With the helical magnet, the spin axis is located approximately 5 degrees away from the longitudinal but still in the horizontal plane. For the entire AGS, the one-turn spin matrix is

$$
M_{\text {spin } A G S}=M_{\text {spin }} M_{d}(-2 \pi)
$$

and the spin tune can be found by using

$$
\nu_{\text {spin }}=\frac{1}{2 \pi} \cos ^{-1}\left(\frac{\operatorname{tr} M_{\text {spinAGS }}-1}{2}\right) .
$$

Since the rotation axis for the partial Siberian Snake lies in the horizontal plane, the spin tune follows

$$
\cos \pi \nu_{\text {spin }}=\cos \frac{\delta}{2} \cos \pi G \gamma
$$

The spin tune versus energy, or equivalently versus $G \gamma$, is shown in Fig. 4 near the $G \gamma=8$ imperfection resonance. At the imperfection resonances the partial snake keeps the spin tune away from the resonance condition.

\section{Coupling due to the Two Snake Options}

The main goal of the helical system is to reduce the amount of transverse coupling introduced by the Partial Snake. Thus, we consider the coupled transverse motion in the presence of each variety of Partial Snake. The $4 \times 4$ orbital transport matrix of the solenoid magnet in the AGS, with central longitudinal field $B_{s}$ and effective length $L_{s}$ can be written as

$$
\begin{aligned}
M_{\text {sol }}= & \left(\begin{array}{cccc}
1 & 0 & 0 & 0 \\
0 & 1 & -K / 2 & 0 \\
0 & 0 & 1 & 0 \\
K / 2 & 0 & 0 & 1
\end{array}\right) \times \\
& \left(\begin{array}{cccc}
1 & \frac{1}{K} \sin \left(K L_{s}\right) & 0 & \frac{1}{K}\left[1-\cos \left(K L_{s}\right)\right] \\
0 & \cos \left(K L_{s}\right) & 0 & \sin \left(K L_{s}\right) \\
0 & -\frac{1}{K}\left[1-\cos \left(K L_{s}\right)\right] & 1 & \frac{1}{K} \sin \left(K L_{s}\right) \\
0 & -\sin \left(K L_{s}\right) & 0 & \cos \left(K L_{s}\right)
\end{array}\right)\left(\begin{array}{cccc}
1 & 0 & 0 & 0 \\
0 & 1 & K / 2 & 0 \\
0 & 0 & 1 & 0 \\
-K / 2 & 0 & 0 & 1
\end{array}\right)
\end{aligned}
$$

where $K \equiv B_{s} / B \rho$. The $4 \times 4$ matrix of the helical dipole magnet can be written as

$$
M_{h e l}=\left(\begin{array}{cccc}
c_{\delta} & 0 & -s_{\delta} & 0 \\
0 & c_{\delta} & 0 & -s_{\delta} \\
s_{\delta} & 0 & c_{\delta} & 0 \\
0 & s_{\delta} & 0 & c_{\delta}
\end{array}\right)\left(\begin{array}{cccc}
c_{0} & s_{0} / \lambda_{0} & 0 & 0 \\
-\lambda_{0} s_{0} & c_{0} & 0 & 0 \\
0 & 0 & c_{0} & s_{0} / \lambda_{0} \\
0 & 0 & -\lambda_{0} s_{0} & c_{0}
\end{array}\right)
$$


Spin tune near $\mathrm{G}$ gamma = integer

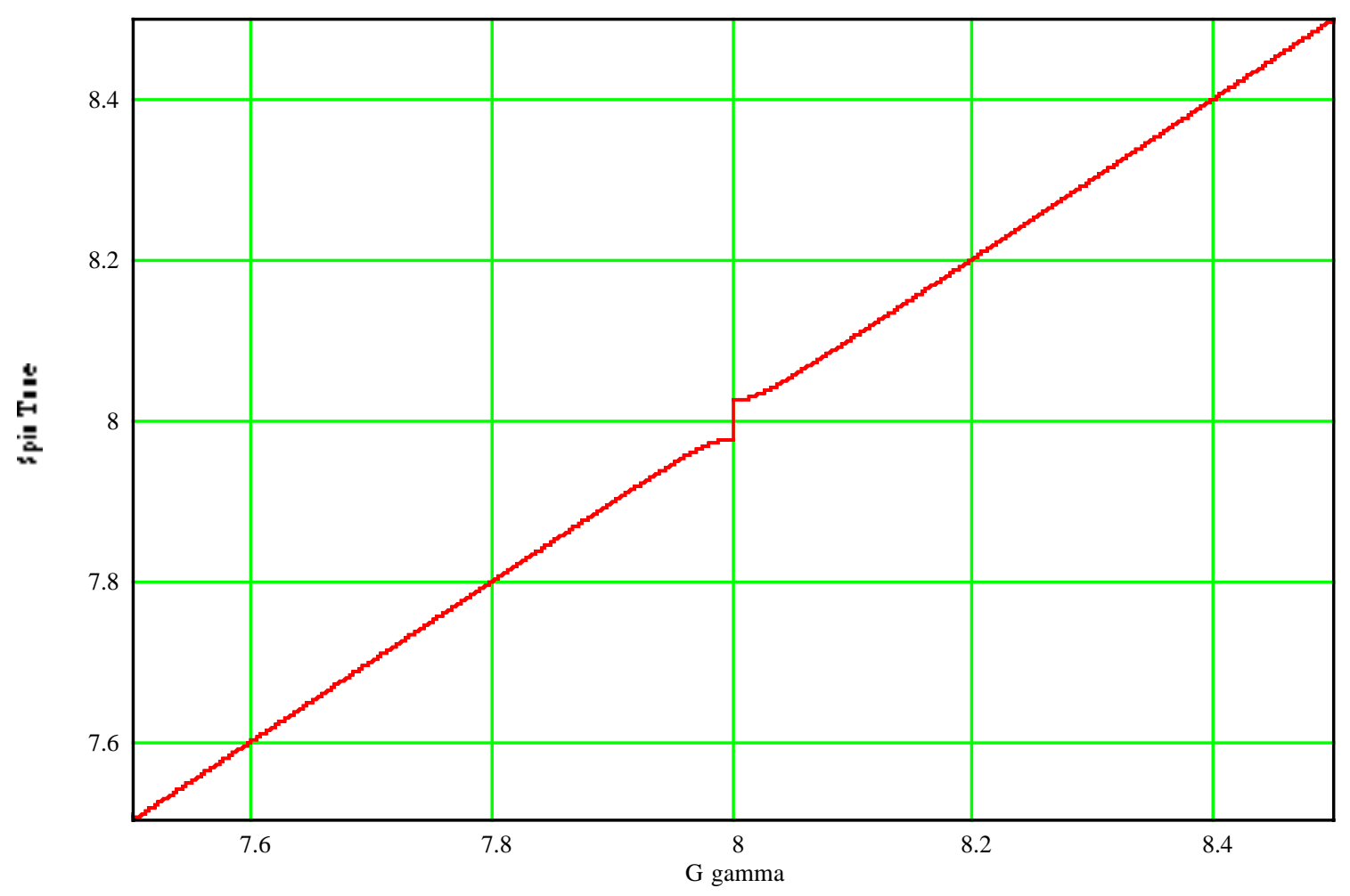

Figure 4: Spin tune versus $G \gamma$ near the imperfection resonance $G \gamma=8$.

where here $c_{\delta} \equiv \cos \delta L_{h}, c_{0} \equiv \cos \lambda_{0} L_{h}, s_{\delta} \equiv \sin \delta L_{h}, s_{0} \equiv \sin \lambda_{0} L_{h}$, and $\delta=$ $B_{0}^{2} /\left[2 k(B \rho)^{2}\right], \lambda_{0}=\sqrt{\delta^{2}+\left(B_{0} / B \rho\right)^{2} / 2}$.

There is a difference in the operation of these two devices, however. The solenoid magnet used as a spin rotator must ramp with beam energy, since the spin motion is governed by a longitudinal magnetic field. However, in the case of the helical magnet, the spin motion is governed by transverse fields, and hence the helix - and bending magnets - are held approximately constant during the entire AGS cycle to assure the same spin rotation at all energies. (The difference being the difference between $1+G \gamma$ and $B \rho \propto \sqrt{\gamma^{2}-1}$, which become a nearly linear relationship at high energies.) A meaningful comparison thus would be to look at the effects at the first strong intrinsic resonance, $G \gamma=\nu_{y} \approx 8.67$. The coupling induced by the solenoid will remain at this level throughout the cycle, while the coupling induced by the helix will be reduced as the inverse of the beam momentum.

Comparing the two matrices directly for the magnet parameters above, and for the parameters of the solenoid magnet at $G \gamma=8.67$, namely $B_{s}=0.37$ Tesla, with 
$L_{s}=2.286 \mathrm{~m}$, the $4 \times 4$ transport matrices for the two devices are

$$
\begin{gathered}
M_{\text {sol }}=\left(\begin{array}{cccc}
0.999 & 2.285 & 0.029 & 0.066 \\
-0.00036 & 0.999 & -0.000010 & 0.029 \\
-0.029 & -0.066 & 0.999 & 2.285 \\
0.000010 & -0.029 & -0.00036 & 0.999
\end{array}\right), \\
M_{\text {hel }}=\left(\begin{array}{cccc}
0.994 & 1.497 & -0.0018 & -0.0028 \\
-0.0077 & 0.994 & 0.000014 & -0.0018 \\
0.0018 & 0.0028 & 0.994 & 1.497 \\
-0.000014 & 0.0018 & -0.0077 & 0.994
\end{array}\right) .
\end{gathered}
$$

We see immediately that some coupling terms for the helix are about 10-20 times smaller than the coupling terms for the solenoid.

To better quantify the effects, we look at the one-turn $4 \times 4$ matrix of the (otherwise uncoupled) AGS in the presence of either the solenoid or the helix, and compute the minimum tune separation, $\Delta \nu_{\text {min }}$, generated by the non-zero coupling terms of the complete matrices. If the $4 \times 4$ orbit matrix of the Snake of length $L_{s n}$ is $M_{s n}$, then the total AGS ring matrix at the exit of the Snake can be written as

$$
M_{A G S}=M_{s n}\left(\begin{array}{cc}
1 & -L_{s n} \\
0 & 1
\end{array}\right) M_{0}
$$

where $M_{0}$ is the $4 \times 4$ matrix of the otherwise uncoupled AGS ring. If the horizontal and vertical tunes of the uncoupled AGS are both set equal to $\nu_{0}$, then the minimum tune separation can be found by

$$
\Delta \nu_{\min } \approx \frac{\sqrt{\operatorname{det}\left(F+E^{\dagger}\right)}}{2 \pi\left|\sin 2 \pi \nu_{0}\right|}
$$

where

$$
M_{A G S}=\left(\begin{array}{cc}
A & E \\
F & B
\end{array}\right)
$$

and $E^{\dagger}$ is the symplectic conjugate of the $2 \times 2$ submatrix $E$.

Assuming that both the horizontal and vertical tunes are set to 8.673 with the solenoid and helix turned off, we find for the two cases where either device is turned on that

$$
\Delta \nu_{\text {minSolenoid }}=0.015, \quad \Delta \nu_{\operatorname{minHelix}}=0.00095
$$

at $G \gamma=8.673$. Thus, the coupling due to the helix is only about $6 \%$ of the coupling due to the solenoid at this energy. The effect only gets better at higher energies, as the coupling due to the helix diminishes.

We see that it should be possible to design a helical dipole magnet system for use as a Partial Siberian Snake in the AGS which would in principle significantly reduce 
the transverse linear coupling and hence improve performance of polarized proton operations. The system would produce roughly a 5\% Partial Snake, composed of a single helical dipole magnet and two short bending dipole magnets, placed within a single 10-foot straight section of the AGS. The maximum orbit excursions at injection would be approximately \pm 1 inch. At higher energies, where the $\mathrm{AC}$ dipole is exercised, the orbit excursions in the Snake are smaller. The beam pipe, of course, must be designed to handle the total excursions.

A preliminary design of a magnet cross section has been produced. A similar design was generated during a study of polarized proton acceleration at Fermilab, where a $10 \%$ scale model was actually constructed and tested[4]. The magnet has a

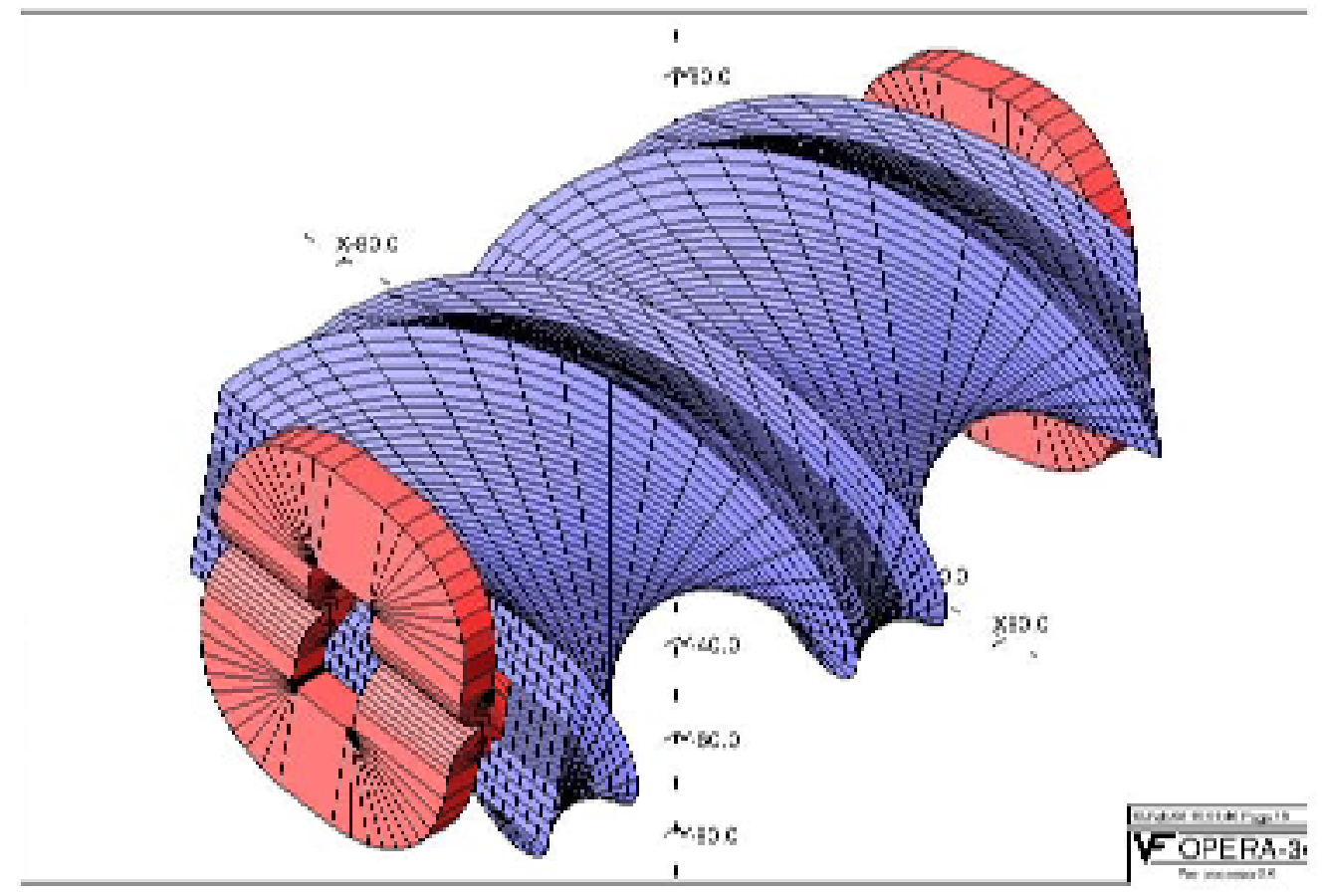

Figure 5: Three dimensional model of possible normal conducting helical dipole magnet to be used as part of Partial Siberian Snake in the AGS.

$15 \times 15 \mathrm{~cm}$ central aperture, 150 turns, and an operating current of about $1100 \mathrm{~A}$, giving a central field of 1.4 Tesla with reasonable multipole content. A 3-dimensional model of the helical magnet is shown in Fig. 5. It is envisaged that the magnet coils and rectangular laminations would gradually and smoothly rotate around the magnet axis along its length, surrounding a circular $15 \mathrm{~cm}$ beampipe.

Much more work needs to be performed on the design. The pitch of $360^{\circ}$ in only 1.5 meters will generate nonlinear fields, as well as strong longitudinal fields off axis. (Note that the beam will travel approximately $25 \mathrm{~mm}$ off the central axis through the 
magnet.) The values of these fields and their effects on the beam will be evaluated

soon. Needless to say, developing the construction technique for such a helical magnet coil will be technically challenging as well.

\section{References}

[1] E.D. Courant, "Hybrid Helical Snakes and Rotators for RHIC," BNL-61920, AD/RHIC-133, AGS/RHIC/SN-010, June 1995.

[2] H. Goldstein, "Classical Mechanics," 2nd Ed., Addison-Wesley (1981), p. 165.

[3] Ibid, p. 172.

[4] "Acceleration of Polarized Protons to $120 \mathrm{GeV}$ and $1 \mathrm{TeV}$ at Fermilab," SPIN Collaboration, U. of Mich. internal report UM HE 95-09, July 1995. 